\title{
A novel metric for community detection
}

\author{
Ke-ke Shang ${ }^{1 *}$, Michael Small ${ }^{2,3}$, Yan Wang ${ }^{1}$, Di Yin ${ }^{1}$, Shu Li ${ }^{1}$, \\ ${ }^{1}$ Computational Communication Collaboratory, Nanjing University, Nanjing, 210093, P.R. China \\ ${ }^{3}$ Mineral Resources, CSIRO, Kensington, WA, 6151, Australia \\ kekeshang@nju.edu.cn, keke.shang.1989@gmail.com
}

${ }^{2}$ Complex Systems Group, Department of Mathematics and Statistics, The University of Western Australia, Crawley, Western Australia 6009, Australia

\begin{abstract}
Research into detection of dense communities has recently attracted increasing attention within network science, various metrics for detection of such communities have been proposed. The most popular metric - Modularity - is based on the so-called rule that the links within communities are denser than external links among communities, has become the default. However, this default metric suffers from ambiguity, and worse, all augmentations of modularity and based on a narrow intuition of what it means to form a "community". We argue that in specific, but quite common systems, links within a community are not necessarily more common than links between communities. Instead we propose that the defining characteristic of a community is that links are more predictable within a community rather than between communities. In this paper, based on the effect of communities on link prediction, we propose a novel metric for the community detection based directly on this feature. We find that our metric is more robustness than traditional modularity. Consequently, we can achieve an evaluation of algorithm stability for the same detection algorithm in different networks. Our metric also can directly uncover the false community detection, and infer more statistical characteristics for detection algorithms.
\end{abstract}

\section{Introduction}

Network substructures - communities - appear in many complex networks and are frequently associated with important functions of those networks (Girvan and Newman 2002; Spirin and Mirny 2003; Krause et al. 2003). Research into community detection has recently attracted increasing attention (Clauset, Newman, and Moore 2004; Li, Ng, and Ye 2013; Chakraborty et al. 2016; Fortunato and Hric 2016). Meanwhile, the design of metrics to evaluate community detection has also been a recent focus of investigation (Newman and Girvan 2004; Fortunato and Barthelemy 2007; Lancichinetti, Fortunato, and Radicchi 2008; Chakraborty et al. 2017). The metrics of community structure can be generally grouped within three classes: (1) based on the density of internal links (links within the community)(Radicchi et al. 2004); (2) based on the density of external links (Radicchi et al. 2004; Fortunato and Hric 2016); and, (3) based on the the density of both internal links and external links (Newman

\footnotetext{
${ }^{*}$ Ke-ke Shang is the corresponding author Copyright (c) 2019, Association for the Advancement of Artificial Intelligence (www.aaai.org). All rights reserved.
}

and Girvan 2004). However, we argue that all these metrics are based on an unnecesary assumption - that the density of internal links must be greater than that of external links. While this is true for communities in many real networks, it is not always the case. Clearly, of course, this depends on what one means by a "community", but we argue here that the natural intuition for community does not necessarily imply increased link density. As depicted in Fig. 1, there are two typical communities in the natural world. Figure 1 (a) shows a mode network for which links within communities are denser than those between groups, however, Fig.1 (b) shows another quit typical community. In this case, Fig. 1 depicts a typical work unit, and the associated subordinate relationships. The community in this case, a common work association, exhibits hierarchical rather than simply dense link structure. Moreover, the nodes group within the same region of geodesic space also can be called as the community (Mahmood and Small 2015; Mahmood et al. 2016). Hence, based on different kinds of real-world communities, we argue an intuitive generalisation of the existing definition of community structure.

\section{(a) Regular Community}

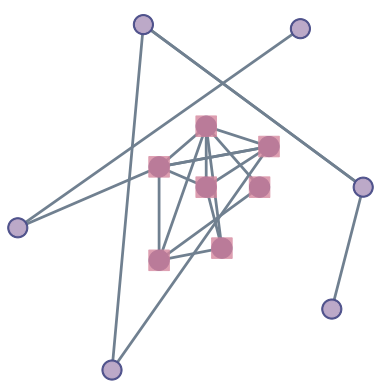

(b) Another Real Community

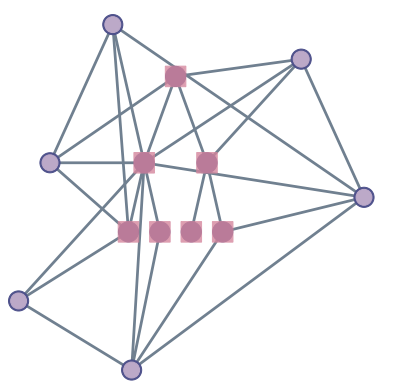

Figure 1: Two different kinds of communities. The squares indicate nodes within a single community. Conversely, the circles indicate nodes external to the community. Panel (a) depicts the traditional community which was been wellstudied by previous studies. Panel (b) is an another realworld ("corporate") community which may (for example) reflect an internal management structure.

More importantly, the default metric Modularity (New- 
man and Girvan 2004; Newman 2006b) and its associated generalisations and extensions (Fortunato and Barthelemy 2007; Chakraborty et al. 2017) are also based on the preceding density assumption. In this paper we focus on the fact that links within a community are more predictable than external links (Yan and Gregory 2012; Cannistraci, Alanis-Lobato, and Ravasi 2013; Ding et al. 2016) - due simply to the assumption that relationships within a real community are stronger (and perhaps more structured) than the external relationships. Hence, we adopt ideas from link prediction theory - where links can be predicted based on statistical characterisation of network structure (LibenNowell and Kleinberg 2007; Redner 2008; Lü et al. 2015; Shang et al. 2019) - to measure the predictability of network substructure. We propose a new metric for community detection which is based on the principle that links within a community should achieve a higher link prediction performance than external links (Yan and Gregory 2012; Cannistraci, Alanis-Lobato, and Ravasi 2013; Ding et al. 2016). Link prediction should work best within communities and worst between communities.

In this paper, we employ 5 famous network open datasets to be tested. First, we use 8 traditional community detection algorithms to uncover the communities, every algorithm has its corresponding communities for each network. Second, we analyze the link prediction performance (predictability) for the internal links and that of all links respectively. Third, we compare the performance of above two kinds of links for each network, and normalize the difference between them as the score of each detection algorithm. Meanwhile, we test the default metric Modularity for each network. We find that our metric is more stable and consistent than Modularity for each detection algorithm in all 5 networks. Our metric can also uncover more statistical rules for each detection algorithm. We suggest that our metric not only can be more widely used, but will also achieve a more stable estimate for each algorithm. Furthermore, our metric can directly expose the failure of current community detection algorithms.

\section{Network data}

As listed in Table 1, we employ 5 open traditional network datasets that have been widely applied for community detection or link prediction problems. (1) Karate club (Zachary 1977): A node represents one club user, a link indicates that there is a relationship between a pair of nodes. Karate club network is the common used data for community detection (Girvan and Newman 2002). (2) Adjectives and nouns (Adjnoun)(Newman 2006a): a node represents one word (adjective or noun), a link indicates that any two words are next to each other in the book. Adjectives and nouns network is also the common used data for community detection (Newman 2006a). (3) Polbooks (Adamic and Glance 2005): a node represents one book about US politics which is sold by Amazon.com, a link stands for there is a pair of nodes are purchased by a same buyer. Polbooks is the common used data for both community detection and link prediction (Adamic and Glance 2005; Shang, Small, and Yan 2017). (4) C.Elegans network (Lü and Zhou 2010): A node represents one neuron, a link indicates a synapse or a gap junction
Table 1: The number of nodes and links, and the source for 5 undirected networks. We do not count the number of loop links and isolated nodes.

\begin{tabular}{cccccc}
\hline \hline & Karate & Adjnoun & Polbooks & C.Elegans & Usair \\
\hline Nodes & 34 & 112 & 105 & 296 & 332 \\
Links & 78 & 425 & 441 & 2148 & 2126 \\
\hline \hline
\end{tabular}

between a pair of nodes. C.Elegans network is the common used data for link prediction (Lü and Zhou 2011; 2010). (5) Usair (Lü and Zhou 2010): A node represents one airport, a link indicates there is a fight line between a pair of nodes. Usair network is also the common used data for link prediction (Lü and Zhou 2011; 2010).

\section{Methods \\ Metric for community detection}

At the start, we use the detection algorithm to uncover the communities of network. Based on the assumption that the internal links within communities are more predictable than external or all links (Yan and Gregory 2012; Cannistraci, Alanis-Lobato, and Ravasi 2013; Ding et al. 2016), we adopt three famous link prediction algorithms to test the predictable of internal links, then get the prediction accuracy scores of internal links $S_{i n}^{1}, S_{i n}^{2}, S_{i n}^{3}$, and those of all links $S_{\text {all }}^{1}, S_{\text {all }}^{2}, S_{\text {all }}^{3}$. Then we can achieve the detection score,

$$
S_{p r}=\frac{\sum_{i=1}^{n} \frac{S_{i n}^{i}-S_{a l l}^{i}}{S_{a l l}^{i}}}{n} .
$$

Particularly, we can adopt the link prediction algorithm for tree-like networks (Shang et al. 2019) if the community structure is similar to the Figure.1 (b). The accuracies of link prediction algorithms for all links $\left(S_{\text {all }}\right)$ will have values in $[0.5,1$ ) except for that of Preferential attachment index (Lü and Zhou 2011; Shang et al. 2019). In addition, theoretically, $S_{i n}$ should higher than $S_{\text {all }}$. Hence, for an effective community detection algorithm, the $S_{p r}$ should values in $(0,1)$ when we do not adopt the Preferential Attachment index ${ }^{1}$. Obviously, the higher value of $S_{p r}$ means the better performance of community detection algorithms. In detail, the negative value of $S_{p r}$ means the disadvantage role of the detected communities for link prediction. However, previous studies show that the community plays an advantageous role for link prediction. That is, the corresponding detection algorithm is failing when $S_{p r} \leq 0$. Generally, the effective detection algorithm should achieve a positive $S_{p r}$ value.

Moreover, we can also extend the metric for overlapping communities. By generalising out previous arguments, we follow the principle that the scores of internal links should bigger than those of overlapping links, and the scores of overlapping links should bigger than those of external links. In this paper, we pay attention to the basic non-overlapping community problem.

\footnotetext{
${ }^{1}$ The AUC of the Preferential attachment index is usually lesser than 0.5 .
} 


\section{Link prediction problem}

The graph $G$ can be completely described by a vertex set $V$, all pair of nodes set $U$, and an edge set $E: G=(V, E)$. The vertex set is stationary and does not evolve. Elements of the edge set are unordered pairs of elements of the vertex set: $e=\left(v_{i}, v_{j}\right) \in E$ where $v_{i}, v_{j} \in V$. The pair $\left(v_{i}, v_{j}\right)$ occurs in at most one edge $e \in E$. The edge set $E$ is divided into two parts $E^{T}$ and $E^{P}$ where $E^{T} \cup E^{P}=E$ and $E^{T} \cap$ $E^{P}=\emptyset$. The division into $E^{P}$ (typically including $10 \%$ of the observed links in (Lü and Zhou 2010)) and $E^{T}$ (typically $90 \%$ of the observed links) is arbitrary and will be used for scoring purposes. The static ${ }^{2}$ link prediction problem can be stated: given the training link set $E^{T}$ and the probe link set $E^{P}$ (and also $V$ ), $E^{\prime \prime}=U \backslash E$, then predict a small part of unobserved links in $E^{\prime \prime}$ and that of fake links in $E^{P}$. That is, if we know some of the links of a network - those links being partitioned into the training set $E^{T}$ and the probe set $E^{P}$ - which we have observed, is it possible to predict the existence (or otherwise) of unobserved or fake links. The unobserved links are members of $E^{\prime \prime}$ and may be said to either exist or be non-existent. Generally, the link prediction scores of existence links are bigger than those of fake links or non-existence links.

Link prediction algorithms The friend of our friend is our friend also, as is figured by a closed triangular structure. This common intuition is the basis of all local link prediction algorithms, with the except of the preferential attachment index. Newman et al. first use this rule to study the cooperation behaviors of scientists (Newman 2001), which then provided a foundation for link prediction problem (Liben-Nowell and Kleinberg 2007). Based on these studies, the well-known common neighbors index $(C N)$ has been proposed:

$$
S_{i j}^{C N}=|\Gamma(i, j)|,
$$

where $\Gamma(i, j)$ denotes the set of common neighbors of the nodes $i$ and $j$. The algorithm indicates that if you have a common friend with another person, there is a possible relationship between you. That is to say the friend (common neighbors) of friend is our friend.

Leicht-Holme-Newman index ( $L H N 1$ ) (Leicht, Holme, and Newman 2006) directly compare the number of common neighbors and the value which is proportional to the possible number of that:

$$
S_{i j}^{L H N 1}=\frac{|\Gamma(i, j)|}{k(i) \times k(j)} .
$$

Hub depressed index (HDI) (Lü and Zhou 2010) is associated with the hub promoted index (HPI) (Ravasz et al. 2002), both of them are based on the role of common neighbors. Hub promoted index aims at the improving of hub nodes effects. On the contrary, hub depressed index aims at the decreasing of hub nodes effects:

$$
S_{i j}^{H D I}=\frac{|\Gamma(i, j)|}{\max \{k(i)+k(j)\}} .
$$

\footnotetext{
${ }^{2}$ And this is all that we consider here.
}

Metric for link prediction algorithms The Area under Receiver Operating Characteristic Curve $(A U C)^{3}$ was originally applied to evaluate communication schemes and has since been widely applied more generally to measure prediction accuracy (Hanley and McNeil 1982). We use $A U C$ as a link prediction accuracy measure for networks. Only the information of $E^{T}$ is allowed to be used to compute the performance score, we compare the prediction scores of $m$ pairs of nodes from $E^{P}$ and $E^{\prime \prime}$ randomly, if there are $m^{\prime}$ times that the score measured from $E^{P}$ is bigger than the score measured from $E^{\prime \prime}$ and $m^{\prime \prime}$ times that the two scores are equal, then, the prediction accuracy $A U C=$ $\left(m^{\prime}+0.5 m^{\prime \prime}\right) / m$. In this letter, we compute the AUC of all algorithms 100 times independently.

\section{Results}

Similar to previous studies (Girvan and Newman 2002), we focus on correctly identifying links within communities. Assuming that links within a community are more predictable than external links, we compare the accuracy of link prediction for internal links and that of all (internal and external) links. Here, we adopt 8 traditional community detection methods to be tested. (1) Kclique algorithm is based on the relationship between nodes and subgraphs (Palla et al. 2005); (2) Fast greedy (FG) adopts the greedy optimization method (Clauset, Newman, and Moore 2004); (3) Girvan-Newman algorithm (GN) is based on the principle of edge betweenness (Girvan and Newman 2002); (4) Label Propagation algorithm (LP) is based on the propagation process of labeled nodes (Xie and Szymanski 2012); (5) the leading eigenvector of modularity matrix is the key for Leading eigenvector algorithm (LE) (Newman 2006b); (6) MultiLevel algorithm actually is the multiresolution version of modularity (Breiger, Boorman, and Arabie 1975); (7) WalkTrap algorithm adopts the random walk method (Pons and Latapy 2005); and (8) InfoMap algorithm is based on random walk dynamics (Rosvall and Bergstrom 2008). These diverse algorithms make it more accurate for analyzing the predictability of community structures.

\section{Internal links versus all links}

As shown in Figure. 2, we can see that, for almost all link prediction algorithms, the prediction accuracies of internal links is higher than that of all links. That is to say, the internal links are more predictable than all links, further than external links. This result also provides the design basis for our community detection metric. We also observe that for the detection algorithms $F G, L E$ and Multilevel, the $C N$ accuracies of internal links are obviously lower than those of all links in the Adjnoun network and the Usair network.

\footnotetext{
${ }^{3}$ With the abscissa measuring the false positive rate, and the ordinate the true positive rate, we can then draw a Receiver Operating Characteristic Curve (ROC). Statistically, the area under the ROC should be between 0.5 and 1 . If the area is greater than 0.5 , we can suggest that our method is effective. If the area equals to 0.5 , then our method is invalid. The case that the area is less than 0.5 , is unrealistic - in this situation the method performs so
} 

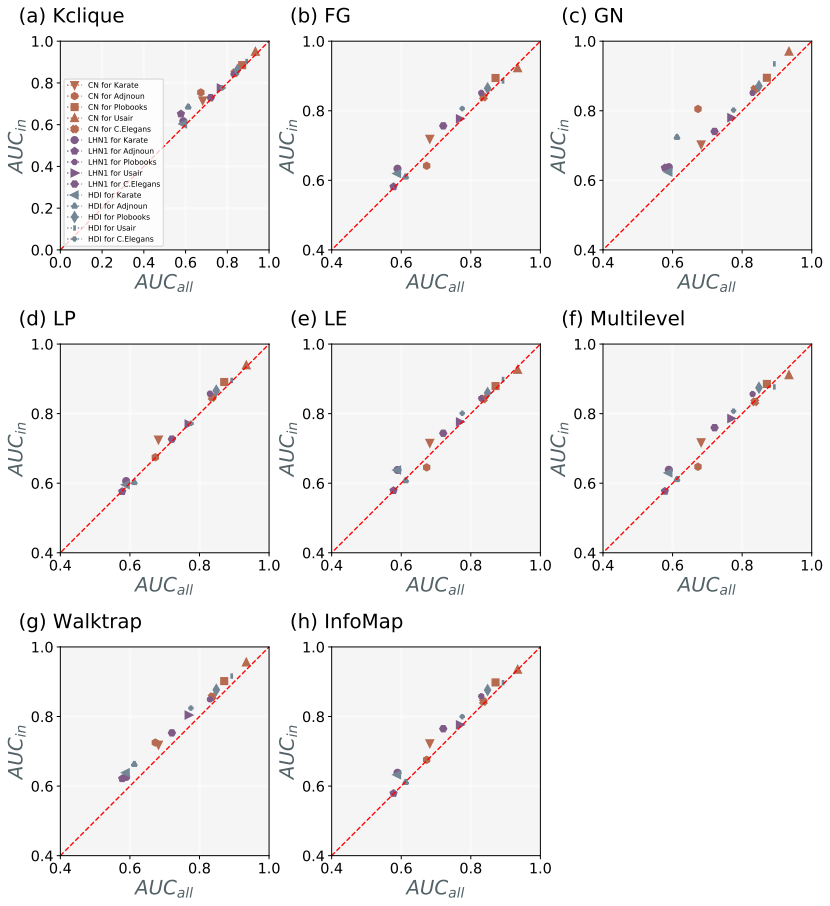

Figure 2: For 8 traditional community detection algorithms, the prediction accuracies of community internal links versus all links via three traditional link prediction algorithms in 5 traditional networks. The ordinate is the AUC score of internal links, and the abscissa is the AUC score of all links. The dashed line is the diagonal. Obviously, the dot which is above the diagonal means the AUC score of internal links is bigger than that of all links for the corresponding link prediction algorithm.
Hence, to clearly distinguish the qualities of detection algorithms. As depicted in Figure. 3, we show the average link prediction accuracies of all three link prediction algorithms for each community detection algorithm. We can clearly see that, in general, the prediction accuracies of internal links are higher than those of all links. In detail, for the algorithms Kclique, Girvan-Newman and WalkTrap, the prediction accuracies of internal links always are higher than that of all links. On the contrary, for algorithms $F G$, $L E$ and Multilevel, the prediction accuracies of internal links are lower than those of all links in the Adjnoun network and the Usair network, namely these community detection algorithms are not effective for the Adjnoun network and the Usair network. (a) Kclique

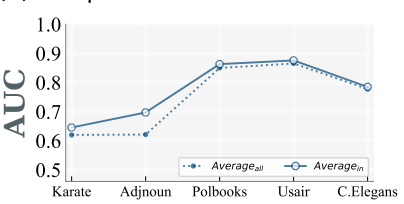

(c) GN

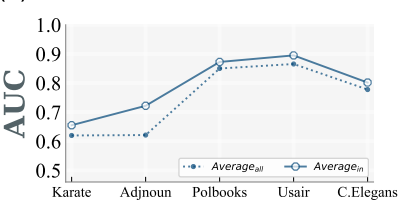

(e) LE

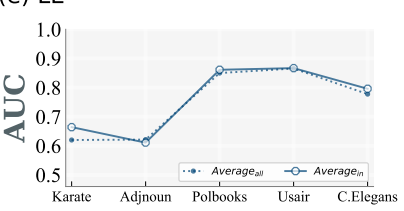

(g) Walktrap

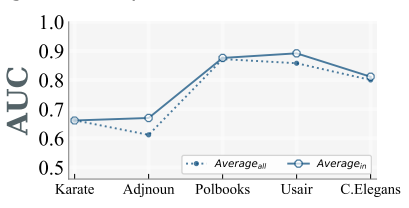

(b) FG

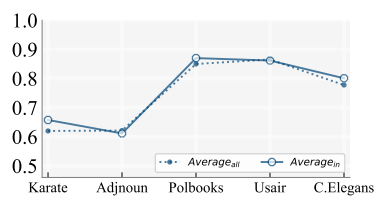

(d) LP

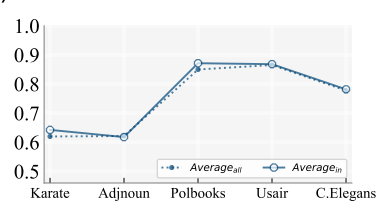

(f) Multileve

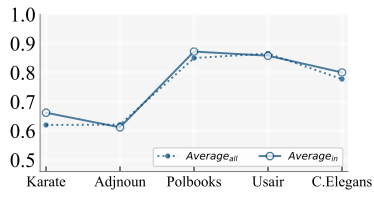

(h) InfoMap

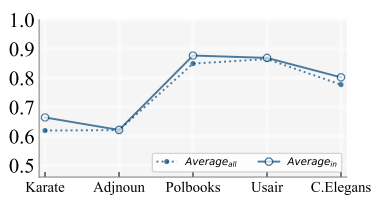

Figure 3: For 8 traditional community detection algorithms, the average prediction accuracies of community internal links and all links of three traditional link prediction algorithms in 5 traditional networks. The hollow dots indicate the results of internal links, and the filled dots indicate the results of all links.

\section{Predictability versus Modularity}

In what follows, we directly compare our metric and the default metric Modularity (Newman and Girvan 2004; Newman 2006b). As shown in Figure. 4 (a), under our metric, we can see that the same community detection algorithm performs similar in different networks. On the contrary, under the default metric Modularity, the performance of

poorly that it would be better to do the reverse of what is predicted. 
the same community detection algorithm varies greatly from network to network (Figure. 4 (b)). Hence, as shown in Table. 2, we adopt the standard deviation to further measure the robustness for these two metrics. The standard deviation of our metric is much less than that of Modularity. Obviously, our metric Predictability is more stable than Modularity. Hence, we infer that the Modularity cannot consistently measure a community detection algorithm in different networks. Our metric does lead to a consistent and stable evaluation for the same community detection algorithm between different networks.
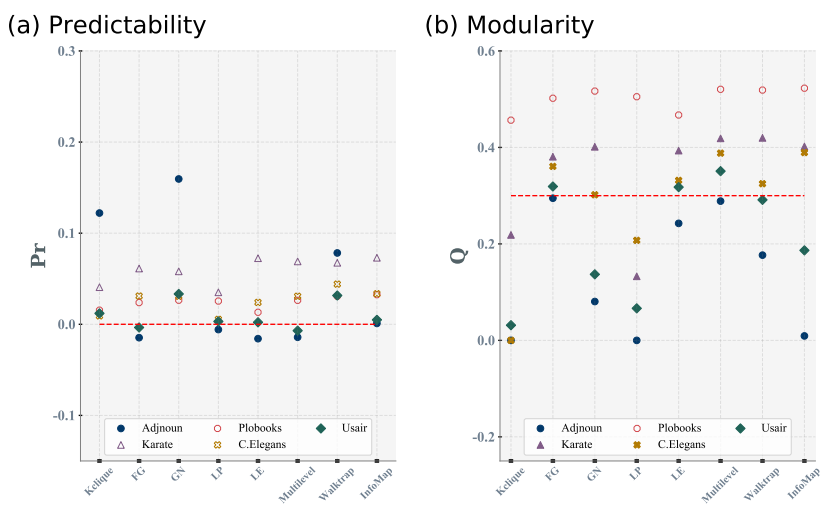

Figure 4: For 5 famous networks, the results of metric Predictability and metric Modularity. The ordinate is the score of corresponding metric. The dashed line indicates the effective score. The hollow marks indicate the results of corresponding networks are more stable than those of other networks.

In addition, our metric can directly and carefully judge the failure of community detection algorithms - if a community detection algorithm achieves a negative Predictability score, we can infer the failure of community detection. For example, as depicted in Figure. 4 (a), for the Adjnoun network and the Usair network, the Fastgreedy algorithm and Multilevel algorithm both achieve the negetive scores, which means that they cannot divide the communities successfully.

More importantly, we also find that the algorithms Kclique, Girvan-Newman and WalkTrap are successful for each network (Figure. 4 (a)). Furthermore, Modularity can indirectly measure the ( $Q \geq 0.3$ (Clauset, Newman, and Moore 2004)) the failure of community detection. However, as shown in Figure. 4 (b), we cannot find any successful community detection algorithm for all networks. We suggest that the weak robustness of Modularity is the key reason for that phenomenon. And our metric Predictability can capture more common statistical characteristics for the community detection algorithms.

As shown in Figure. 4 (b), we can find that the results of the Polbooks network are more stable than other networks, which means that Modularity also can capture the common statistical characteristic for the community detection algorithm. However, as shown in Figure. 4 (a), we not only find the results of Polbooks network are stable and always bigger than 0, but also observe that both the results of Karate network and C.Elegans network are stable and always bigger than 0 . That phenomenon further suggest that our metric can capture more common statistical rules for community detection algorithms. Our metric can find more networks that can easy be divided by the community algorithm.

\section{Conclusion and Discussion}

Though there are many competing metrics with which to evaluate community detection, the different statistical characteristics between internal links of communities and external links of communities is always the key principle. In this paper, we argue that the principle that the density of internal links is higher than that of external links, is not a universal characteristic of all "communities" for all realworld networks. Hence, we propose the Predictability metric which is based on the principle that internal links are more predictable than external links. We find that our metric can reliably evaluate the performance of the same community detection algorithms across a range of different networks, however, the default metric Modularity is rather unstable. We infer that the unstable performance of Modularity is due to the ill-defined of communities: the density of links within communities is higher than that of external links.

Furthermore, our metric can evaluate the performance of community detection algorithm more carefully - the failure of community detection can be uncovered and more statistical rules can be found by our metric. In detail, the negative score of Predictability directly reflects the bad or failure level of a community detection algorithm, inversely, the positive score of Predictability reflects the good level of a community detection algorithm. We also can find more networks that can be divided by the community algorithm. We suggest that the different predictabilities between internal links and external links provide more information than the traditional principle of community detection metrics, our metric can help researchers further discuss the precise definition of the community detection problem.

\section{References}

Adamic, L. A., and Glance, N. 2005. The political blogosphere and the 2004 us election: divided they blog. In Proceedings of the 3rd International Workshop on Link Discovery, 36-43. ACM.

Breiger, R. L.; Boorman, S. A.; and Arabie, P. 1975. An algorithm for clustering relational data with applications to social network analysis and comparison with multidimensional scaling. Journal of Mathematical Psychology 12(3):328-383.

Cannistraci, C. V.; Alanis-Lobato, G.; and Ravasi, T. 2013. From link-prediction in brain connectomes and protein interactomes to the local-community-paradigm in complex networks. Scientific Reports 3:1613.

Chakraborty, T.; Kumar, S.; Ganguly, N.; Mukherjee, A.; and Bhowmick, S. 2016. Genperm: a unified method for detecting non-overlapping and overlapping communities. 
Table 2: For all networks, the standard deviations of Predictability metric and Modularity metric.

\begin{tabular}{cccccc}
\hline \hline Standard deviation & Karate & Adjnoun & Polbooks & C.Elegans & Usair \\
\hline Predictability & 0.0135 & 0.0663 & 0.0063 & 0.0120 & 0.0142 \\
Modularity & 0.1012 & 0.1212 & 0.2389 & 0.1217 & 0.1163 \\
\hline \hline
\end{tabular}

IEEE Transactions on Knowledge and Data Engineering 28(8):2101-2114.

Chakraborty, T.; Dalmia, A.; Mukherjee, A.; and Ganguly, N. 2017. Metrics for community analysis: A survey. $A C M$ Computing Surveys (CSUR) 50(4):54.

Clauset, A.; Newman, M. E.; and Moore, C. 2004. Finding community structure in very large networks. Physical Review E 70(6):066111.

Ding, J.; Jiao, L.; Wu, J.; and Liu, F. 2016. Prediction of missing links based on community relevance and ruler inference. Knowledge-Based Systems 98:200-215.

Fortunato, S., and Barthelemy, M. 2007. Resolution limit in community detection. Proceedings of the National Academy of Sciences 104(1):36-41.

Fortunato, S., and Hric, D. 2016. Community detection in networks: A user guide. Physics Reports 659:1-44.

Girvan, M., and Newman, M. E. 2002. Community structure in social and biological networks. Proceedings of the National Academy of Sciences 99(12):7821-7826.

Hanley, J. A., and McNeil, B. J. 1982. The meaning and use of the area under a receiver operating characteristic (roc) curve. Radiology 143(1).

Krause, A. E.; Frank, K. A.; Mason, D. M.; Ulanowicz, R. E.; and Taylor, W. W. 2003. Compartments revealed in food-web structure. Nature 426(6964):282.

Lancichinetti, A.; Fortunato, S.; and Radicchi, F. 2008. Benchmark graphs for testing community detection algorithms. Physical Review E 78(4):046110.

Leicht, E. A.; Holme, P.; and Newman, M. E. 2006. Vertex similarity in networks. Physical Review E 73(2):026120.

Li, X.; Ng, M. K.; and Ye, Y. 2013. Multicomm: Finding community structure in multi-dimensional networks. IEEE Transactions on Knowledge and Data Engineering 26(4):929-941.

Liben-Nowell, D., and Kleinberg, J. 2007. The linkprediction problem for social networks. Journal of the American Society for Information Science and Technology 58(7):1019-1031.

Lü, L., and Zhou, T. 2010. Link prediction in weighted networks: The role of weak ties. EPL 89(1):18001.

Lü, L., and Zhou, T. 2011. Link prediction in complex networks: A survey. Physica A: Statistical Mechanics and its Applications 390(6):1150-1170.

Lü, L.; Pan, L.; Zhou, T.; Zhang, Y.-C.; and Stanley, H. E. 2015. Toward link predictability of complex networks. Proceedings of the National Academy of Sciences 112(8):2325-2330.
Mahmood, A., and Small, M. 2015. Subspace based network community detection using sparse linear coding. IEEE Transactions on Knowledge and Data Engineering 28(3):801-812.

Mahmood, A.; Small, M.; Al-Maadeed, S. A.; and Rajpoot, N. 2016. Using geodesic space density gradients for network community detection. IEEE Transactions on Knowledge and Data Engineering 29(4):921-935.

Newman, M. E., and Girvan, M. 2004. Finding and evaluating community structure in networks. Physical Review E 69(2):026113.

Newman, M. E. 2001. Clustering and preferential attachment in growing networks. Physical Review E 64(2):025102.

Newman, M. E. 2006a. Finding community structure in networks using the eigenvectors of matrices. Physical Review E 74(3):036104.

Newman, M. E. 2006b. Modularity and community structure in networks. Proceedings of the National Academy of Sciences 103(23):8577-8582.

Palla, G.; Derényi, I.; Farkas, I.; and Vicsek, T. 2005. Uncovering the overlapping community structure of complex networks in nature and society. Nature 435(7043):814.

Pons, P., and Latapy, M. 2005. Computing communities in large networks using random walks. In International Symposium on Computer and Information Sciences, 284293. Springer.

Radicchi, F.; Castellano, C.; Cecconi, F.; Loreto, V.; and Parisi, D. 2004. Defining and identifying communities in networks. Proceedings of the National Academy of Sciences 101(9):2658-2663.

Ravasz, E.; Somera, A. L.; Mongru, D. A.; Oltvai, Z. N.; and Barabási, A.-L. 2002. Hierarchical organization of modularity in metabolic networks. Science 297(5586):1551-1555.

Redner, S. 2008. Networks: teasing out the missing links. Nature 453(7191):47-48.

Rosvall, M., and Bergstrom, C. T. 2008. Maps of random walks on complex networks reveal community structure. Proceedings of the National Academy of Sciences 105(4):1118-1123.

Shang, K.-k.; Li, T.-c.; Small, M.; Burton, D.; and Wang, Y. 2019. Link prediction for tree-like networks. Chaos: An Interdisciplinary Journal of Nonlinear Science 29(6):061103.

Shang, K.-k.; Small, M.; and Yan, W.-s. 2017. Fitness networks for real world systems via modified preferential attachment. Physica A: Statistical Mechanics and its Applications 474:49-60. 
Spirin, V., and Mirny, L. A. 2003. Protein complexes and functional modules in molecular networks. Proceedings of the National Academy of Sciences 100(21):12123-12128.

Xie, J., and Szymanski, B. K. 2012. Towards linear time overlapping community detection in social networks. In Pacific-Asia Conference on Knowledge Discovery and Data Mining, 25-36. Springer.

Yan, B., and Gregory, S. 2012. Finding missing edges in networks based on their community structure. Physical Review E 85(5):056112.

Zachary, W. W. 1977. An information flow model for conflict and fission in small groups. Journal of Anthropological Research 33(4):452-473.

\section{Acknowledgements}

Ke-ke Shang is supported by National Natural Science Foundation of China 61803047 and Tencent Research Institute. Michael Small is supported by ARC Discovery Project DP180100718.

\section{Author contributions statement}

Ke-ke Shang conceived the experiments, Yan Wang conducted the experiments, Ke-ke Shang, Michael Small, Yan Wang and Di Yin analyzed the results, Ke-ke Shang, Michael Small and Shu Li wrote the manuscript. All authors reviewed the manuscript.

\section{Competing interests}

The authors declare no competing interests.

\section{Data Availability}

The datasets generated and/or analysed in this study are available from the corresponding author on reasonable request. 